\title{
Qualidade das anotações de enfermagem em unidade de terapia intensiva de um hospital universitário
}

\author{
The quality of nursing notes in an intensive care unit of a university hospital
}

Calidad de las notas de enfermería en unidad de terapia intensiva de hospital universitario

Fabiane Gorni Borsato ${ }^{1}$, Mariana Ângela Rossaneis², Maria do Carmo Fernandez Lourenço Haddad³ Marli Terezinha Oliveira Vannuchi ${ }^{4}$, Dagmar Willamowius Vituri ${ }^{5}$

\section{RESUMO}

As anotações de enfermagem constituem fonte de informações assistenciais que permitem comunicação multiprofissional. Objetivou-se neste trabalho avaliar a qualidade das anotações de enfermagem em terapia intensiva de um hospital universitário. Realizou-se pesquisa descritivo-quantitativa com coleta dos dados secundários, de relatórios da avaliação da qualidade das anotações de enfermagem realizada em 2009. Os resultados dos relatórios foram comparados segundo os critérios completo, incompleto, não preenchido e incorreto, para os quais se adotou como satisfatório acima de $80 \%$, abaixo de $15 \%$, abaixo de $5 \%$ e $0 \%$, respectivamente. Identificaram-se valores satisfatórios para a existência de uma anotação por turno, registros de cuidados inclusive pré e pós-operatórios/exames. Houve queda nos valores de registro de sinais e sintomas, intercorrências e resposta a prescrição de enfermagem. Quanto à estética textual, identificação do autor e checagem de itens, os resultados foram insatisfatórios. Os resultados mostraram diversas inadequações, necessidade de medidas educativas e aprimoramento técnico-assistencial dos profissionais.

Descritores: Enfermagem; Cuidados de Enfermagem; Gestão de Qualidade; Registros de Enfermagem.

\section{ABSTRACT}

Nursing notes are sources of information that allow for multiprofessional communication. The objective of this study was to evaluate the quality of the nursing notes in the intensive care unit of a university hospital. A descriptive-quantitative study was performed utilizing secondary data collection from quality assessment records of nursing notes from 2009. The report results were compared according to the following criteria: complete, incomplete, blank and incorrect, considering, respectively, a satisfactory score of more than $80 \%$, below $15 \%$, less than $5 \%$, and $0 \%$. Satisfactory scores were found for cases involving at least one note per shift and including pre and post-operative care/exams. There was a reduction in the scores for signs and symptoms, complications and response to the nursing care. Regarding textual esthetics, results were unsatisfactory for author identification and item checklists. Results revealed several inadequacies, proving the need for educational measures, and the improvement of nurses' skills and service.

Descriptors: Nursing; Nursing Care; Quality Management; Nursing Records.

\section{RESUMEN}

Las notas de enfermería constituyen una fuente de información asistencial que permite comunicación multiprofesional. Se objetivó evaluar la calidad de las notas de enfermería en terapia intensiva de un hospital universitario. Investigación descriptiva, cuantitativa, con recolección de datos secundarios desde informes de evaluación de calidad de notas de enfermería realizada por la institución en 2009. Los resultados de los informes fueron comparados según los criterios completo, incompleto, no efectuado e incorrecto; se tomó como satisfactorio: sobre $80 \%$, bajo $15 \%$, bajo $5 \%$ y $0 \%$ respectivamente. Se identificaron valores satisfactorios para la existencia de una nota por turno. Hubo una caída en los valores de registro de signos y síntomas, variaciones y respuesta a prescripciones de enfermería. Respecto de la estética textual, identificación del autor y chequeo de ítems, los resultados fueron insatisfactorios. Se evidenciaron diversas inadecuaciones, necesidad de medidas educativas y mejora técnico-asistencial de los profesionales.

Descriptores: Enfermería; Atención de Enfermería; Gestión de Calidad; Registros de Enfermería.

\footnotetext{
${ }^{1}$ Enfermeira. Discente do Programa de Mestrado Acadêmico do Departamento de Enfermagem da Universidade Estadual de Londrina (UEL). Enfermeira do serviço de controle de qualidade em enfermagem do Hospital Dr. Anísio Figueiredo. Londrina, PR, Brasil. E-mail: fabigorni@hotmail.com.

${ }^{2}$ Enfermeira. Discente do Programa de Enfermagem Fundamental (PPGEF) da Escola de Enfermagem de Ribeirão Preto (EERP) da Universidade de São Paulo (USP), nível Mestrado. Londrina, PR, Brasil. E-mail: marianaangela08@hotmail.com.

3 Enfermeira, Doutora em Enfermagem. Professor Associado do Departamento de Enfermagem/UEL. Londrina, PR, Brasil. E-mail: haddad@sercomtel.com.br.

4 Enfermeira, Doutora em Saúde Pública. Professor Associado do Departamento de Enfermagem/UEL. Londrina, PR, Brasil. E-mail: vannuchi@sercomtel.com.br.

${ }^{5}$ Enfermeira, Mestre em Enfermagem. Discente do PPGEF/EERP/USP, nível Doutorado. Enfermeira do Hospital Universitário Regional do Norte do Paraná/UEL. Londrina, PR, Brasil. E-mail: dagmar@uel.br.
} 


\section{INTRODUÇÃO}

A busca pela qualidade nos serviços de saúde passou por um processo evolutivo segundo as alterações sociais, políticas e econômicas ocorridas nas últimas décadas, incorporando novos conceitos. A qualidade em saúde e em especial, na enfermagem, é caracterizada pela obtenção de benefícios e redução de riscos subsidiados por três grandes vertentes: estrutura, processo e resultados ${ }^{(1)}$.

Um dos grandes avanços da enfermagem contemporânea foi o surgimento do Processo de Enfermagem (PE), proposto por Wanda Horta como um modelo de organização da assistência de enfermagem em seis etapas: histórico, diagnóstico, plano assistencial, plano de cuidados ou prescrição de enfermagem, evolução e prognóstico de enfermagem. Esta forma de estruturação do cuidado permitiu e permite até hoje que o profissional enfermeiro organize sua assistência de maneira sistematizada e proceda ao registro de informações oriundas da mesma ${ }^{(2)}$.

Historicamente, denota-se a presença de registros hospitalares desde o Antigo Egito, revelando a presença de documentação há mais de dois mil anos. Na Itália, em 1580, Camilo de Lellis exigiu, de forma sistemática, que existissem, dentre os registros referentes ao cliente, as anotações de enfermagem de cada plantão, marcando o surgimento dos registros formais da categoria(3).

Uma parcela significativa dos registros de enfermagem é constituída por anotações relativas a assistência prestada, realizadas pelos profissionais de enfermagem no ato de suas atividades. São consideradas fontes escritas de informações relativas às condições do paciente, aos cuidados prestados e aos resultados oriundos do mesmo ${ }^{(4)}$.

Uma taxa de $50 \%$ das informações referentes às condições do cliente e ao cuidado prestado ao mesmo são fornecidas pela enfermagem. Desta forma, espera-se que as anotações realizadas por esta categoria permitam a comunicação entre todos os membros da equipe multiprofissional transmitindo informações que facilitem o planejamento, tomada de decisões e continuidade da assistência prestada(5).

Trata-se aqui da comunicação em seu sentido integral como troca de informações que permite a compreensão de quem às recebe. No ambiente hospitalar esta troca é intensa e constante, em especial no processo de assistência, em que o estado do paciente e os cuidados prestados a ele devem ser transmitidos entre os turnos para que toda a equipe tenha conhecimento das ações ${ }^{(6)}$.

Considerando anotações redigidas com fidedignidade, torna-se possível avaliar a assistência prestada(4). O termo avaliação transmite a idéia de julgamento de algo tendo como referência uma escala de valores. Faz-se necessária a busca por dados quanti e qualitativos e análise dos mesmos com base em critérios previamente definidos. Não significa apenas medir, mas também tratar os dados de maneira interpretativa ${ }^{(7)}$. Nos processos avaliativos, a utilização de indicadores como componentes de instrumentos de avaliação permite uma maneira sintética e fácil de levantamento dos dados e dá maior significado no que se pretende avaliar ${ }^{(1)}$.

Neste contexto que envolve as dificuldades em se alcançar a qualidade das anotações de enfermagem como forma de possibilitar comunicação efetiva entre os membros de uma equipe multiprofissional, optou-se por realizar um estudo que deriva de uma pesquisa maior, em que foi analisada a qualidade das anotações de enfermagem em diversas unidades da instituição em estudo. Visto que foram encontrados valores insatisfatórios nas Unidades de Terapia Intensiva e por esta constituir um local de cuidados críticos e de controle intensivo dos pacientes, optou-se por realizar um estudo minucioso identificando os nós-críticos que envolvem os registros de enfermagem na referida unidade.

Desta maneira, esta pesquisa tem como objetivo avaliar a qualidade das anotações de enfermagem em unidades de terapia intensiva de um hospital público de ensino.

\section{MÉTODOS}

Trata-se de um estudo retrospectivo, descritivo de caráter quantitativo, resultante de um estudo maior em que foram identificados resultados insatisfatórios e fragilidades nos registros de enfermagem na Unidade de Terapia Intensiva de um hospital universitário público.

A coleta de dados foi realizada a partir de três relatórios resultantes da auditoria das prescrições de enfermagem realizada pela Assessoria de Controle da Qualidade da Assistência de Enfermagem (ACQAE) da instituição em estudo no ano de 2009. 
A referida assessoria realiza a auditoria das prescrições de enfermagem com o auxílio de um instrumento específico que aborda seis itens sendo um deles "anotações de enfermagem". Este item contém subitens que representam as questões norteadoras para a coleta de dados. Cada subitem está sujeito à avaliação segundo quatro critérios - 1-completo 2- incompleto 3não-preenchido 4- incorreto. Os resultados referentes a cada critério estão na forma de percentual de positividade em relação ao padrão de qualidade das atividades de enfermagem (tabela 1).

Tabela 1: Percentuais de satisfação utilizados na avaliação da qualidade da assistência de enfermagem da ACQAE de um hospital universitário público, para cada critério de avaliação, Londrina, PR, 2009.

\begin{tabular}{ccccc}
\hline \multirow{2}{*}{ Grau de satisfação } & \multicolumn{4}{c}{ Critérios de avaliação } \\
\cline { 2 - 5 } & Completo & Incompleto & Não-Preenchido & Incorreto \\
\hline Satisfatório & Acima de $80 \%$ & Abaixo de $15 \%$ & Abaixo de $5 \%$ & $0 \%$ \\
Insatisfatório & Abaixo de $80 \%$ & Acima de $15 \%$ & Acima de $5 \%$ & Acima de $0 \%$ \\
\hline
\end{tabular}

Estes mesmos percentuais de positividade foram utilizados neste estudo a fim de otimizar a avaliação segundo os objetivos propostos.

Tanto o instrumento quanto a metodologia de avaliação estão subsidiados em estudos que tratam da temática ${ }^{(8-9)}$ adaptados por Haddad(10).

Os dados foram tabulados e analisados no programa Excel e estão apresentados na forma de percentuais por meio de gráficos comparativos referentes aos três meses analisados.

Esta pesquisa foi aprovada pelo Comitê de Ética em Pesquisa com seres humanos da Universidade Estadual de Londrina com o parecer número 063/09 e CAAE $n^{\circ}$ 1182.0.000.268-09.

\section{RESULTADOS}

Foram avaliados, de forma comparativa, com ênfase na evolução da qualidade dos registros, os resultados relativos a três coletas realizadas nos anos de 2009, analisando-se 13 subitens/questões norteadoras.
Os subitens "Existe uma anotação descritiva de alta, transferência ou transportes?" e "As anotações evidenciam a participação da família nos cuidados de pacientes com acompanhante?" não permitiam análise uma vez que todos os resultados foram classificados como não aplicáveis no momento da coleta de dados.

Os resultados permitiram identificar valores satisfatórios no que tange a existência de anotações de enfermagem, nem que esta se limitasse a pelo menos uma a cada período de seis horas como mostra o gráfico 1. Todas as anotações de enfermagem evidenciavam prestação de cuidados variando de 83 a $90 \%$ delas completas e apenas de 7 a $17 \%$ incompletas no ano analisado; todas elas corretas sendo indicado pelo valor $0 \%$ do critério incorreto. Na três coletas, observou-se que $100 \%$ dos documentos de pacientes que foram submetidos a algum procedimento cirúrgico, evidenciavam cuidados pré e pós-operatórios.

Gráfico 1: Comparativo entre os percentuais das três coletas segundo critérios de avaliação no que tange a existência de pelo menos uma anotação de enfermagem no período de 6 horas, em Unidades de Terapia Intensiva de um Hospital Universitário, 2009.

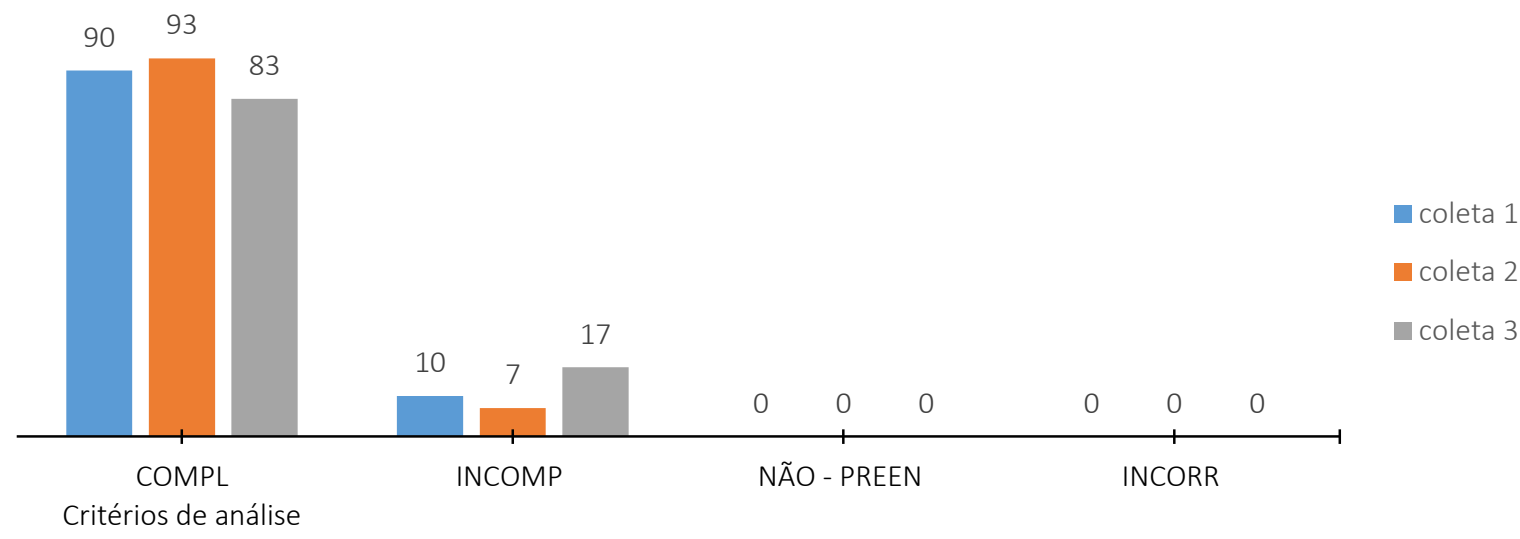


Apesar dos resultados satisfatórios apresentados acima, as anotações de enfermagem apresentaram adequações quanto à existência de intercorrências com os pacientes durante os turnos de trabalho, com $100 \%$ completo das anotações completas nas duas primeiras coletas, evoluindo com queda para $79 \%$ na terceira.
O gráfico 2 apresenta valor positivo relacionado à presença de respostas aos itens da prescrição realizada pelo enfermeiro na primeira coleta. Porém estes valores não foram mantidos, caindo para percentuais abaixo do recomendado na segunda e terceira coleta.

Gráfico 2: Comparativo entre os percentuais das três coletas segundo critérios de avaliação no que tange as respostas das anotações aos itens da prescrição, em Unidades de Terapia Intensiva de um Hospital Universitário, 2009.

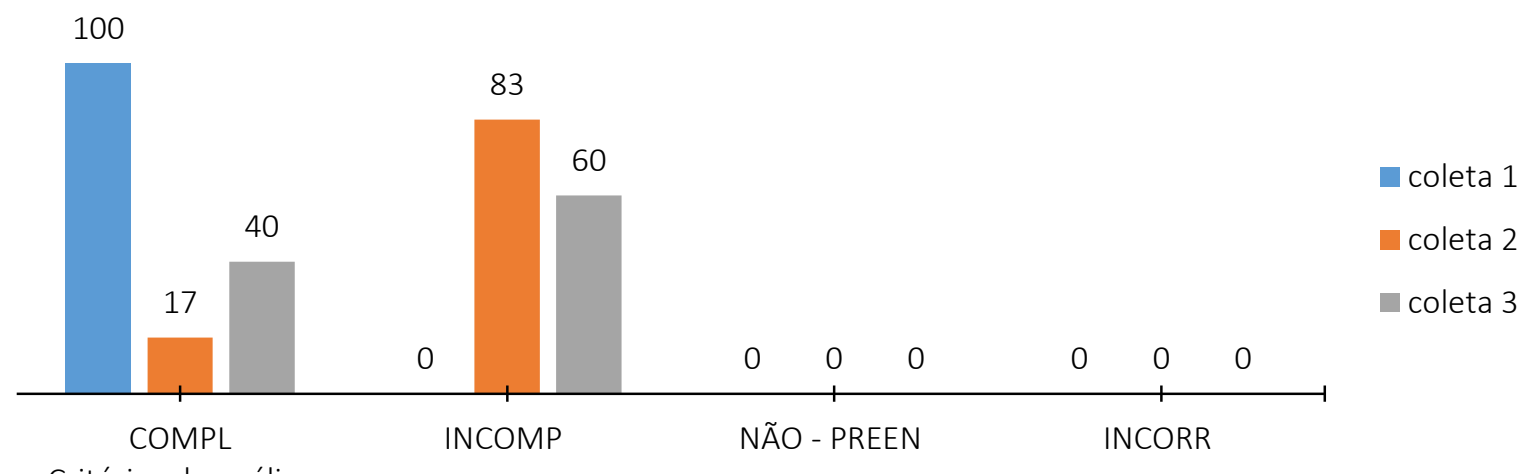

Critérios de análise

O gráfico 3 mostra a alta qualidade nos registros na primeira e segunda coleta com relação à observação do paciente segundo sua patologia e sinais de alteração oriunda dela, valor que não se manteve a partir da terceira coleta, caindo para $33 \%$ de anotações completas. Mesmo que tenham sido encontrados valores insatisfatórios referentes a presença de sinais e sintomas, os registros existentes não apresentavam-se incorretos.

Gráfico 3: Comparativo entre os percentuais das três coletas segundo critérios de avaliação no que tange a existência de anotações que evidenciam a observação de sinais e sintomas pertinentes a patologia, em Unidades de Terapia Intensiva de um Hospital Universitário, 2009.

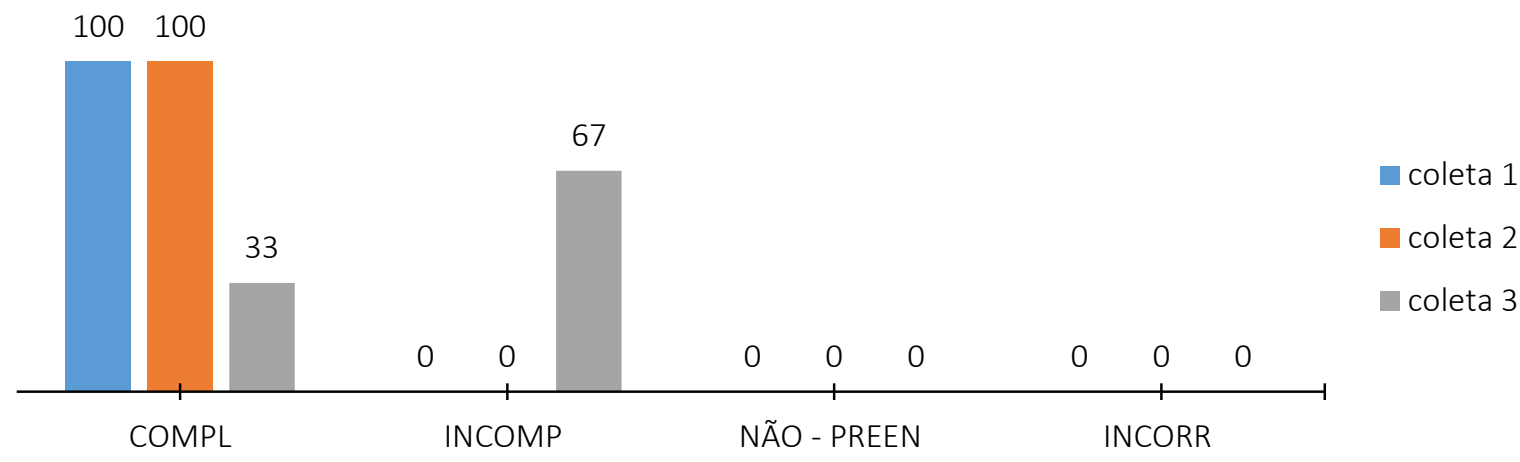

Critérios de análise

Houve uma queda nos percentuais de qualidade no que tange a presença de anotações referentes às condições emocionais do paciente caindo de 100 para $33 \%$.

O estudo mostra um declínio nos percentuais relacionados à utilização de corretor líquido, etiquetas e rasuras nas anotações de enfermagem. Em 100\% das anotações não existem abreviaturas não-padronizadas.

Houve queda progressiva na qualidade das identificações de quem realizou o cuidado e os registrou mantendo-se com números insatisfatórios da primeira a terceira coleta (gráfico 4). 
Gráfico 4: Comparativo entre os percentuais das três coletas segundo critérios de avaliação no que tange a existência de indicação de quem realizou o cuidado (enfermeiro, auxiliar ou técnico de enfermagem), em Unidades de Terapia Intensiva de um hospital universitário, 2009.

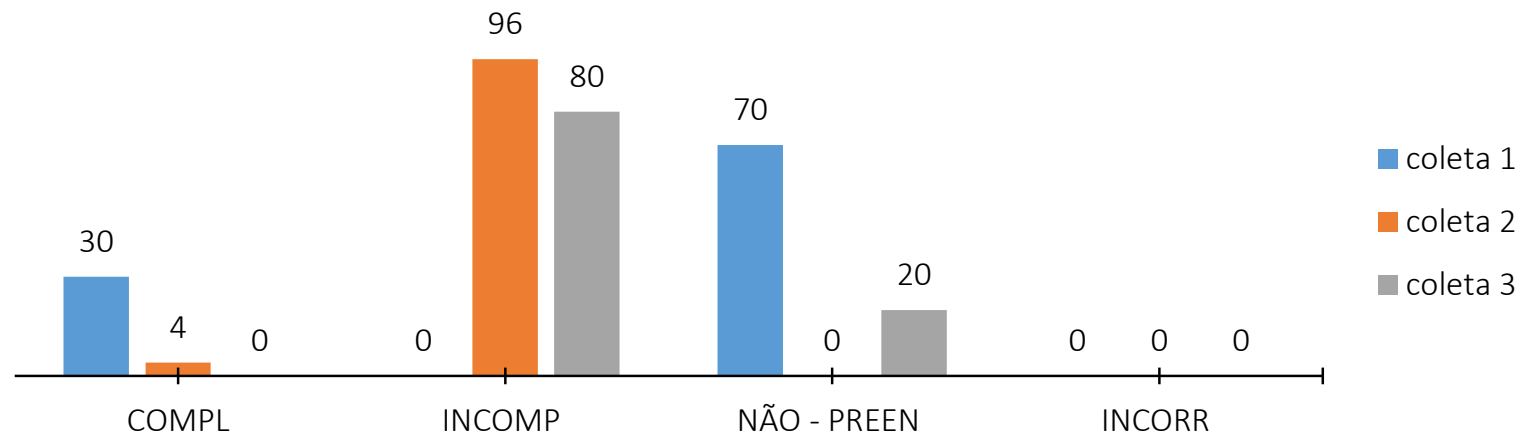

Critérios de análise

Em relação a checagem dos itens prescritos, existem valores insatisfatórios nas três coletas analisadas variando de 7 a $47 \%$ de prescrições de enfermagem com todos os itens checados, não atingido $80 \%$ de satisfação como é preconizado.

Quanto a presença da descrição do aspecto das lesões cutâneas, os valores mantiveram-se satisfatórios nas duas primeiras coletas, baixando para $74 \%$ do item completo e aumento para $22 \%$ do item não-preenchido na terceira coleta.

\section{DISCUSSÃO}

A UTI da instituição em estudo conta com uma equipe de enfermagem capacitada técnica e cientificamente e clientes de alta complexidade clínica, submetidos a diversas intervenções terapêuticas e monitorização por meio de equipamentos de alta tecnologia, e propensos a freqüentes instabilidades clínicas. Neste sentido, é imprescindível a presença de registros eficazes e que contemplem, com fidedignidade, informações relativas as condições do cliente, ações de cuidado e intercorrências ocorridas.

Considerando que esta unidade não permite a participação da família nos cuidados ao paciente, exceto em casos extraordinários, não foi possível avaliar este subitem para a unidade em estudo. Outro ponto a ser destacado foi à impossibilidade de se avaliar anotações descritivas de alta, transferência ou transportes pelo fato de que até o momento da avaliação realizada pelos estagiários da ACQAE, os pacientes não terem sido submetidos a estes procedimentos.
Observou-se a existência de valores satisfatórios em relação a existência de pelo menos uma anotação de enfermagem no período de seis horas, constando, de forma completa, informações relativas a prestação de cuidados, inclusive pré e pós-operatórios quando existirem. Isto denota que o setor em estudo tem, na execução dos registros de enfermagem, a segurança de que informações sejam transmitidas fornecendo subsídios para intervenções de outros profissionais da equipe multidisciplinar ${ }^{(11)}$.

Pesquisa realizada em um hospital universitário na cidade de Curitiba, Paraná, encontrou resultados semelhantes aos da presente pesquisa no que se refere existência de pelo menos uma anotação no período de seis horas. Na pesquisa citada, de todas as unidades avaliadas, apenas uma delas não apresentou anotação de enfermagem relativa aos cuidados com um paciente no período vespertino ${ }^{(3)}$.

Apesar de terem sido encontrados valores satisfatórios quanto a presença de anotações de enfermagem, a pesquisa apresentou queda na qualidade dos registros de intercorrências com o paciente durante o turno. Considerando que as intercorrências são freqüentes em unidades como a estudada neste trabalho em virtude de instabilidades dos pacientes críticos, devese preceder ao relato as mesmas visando a transmissão fidedigna e isenta de vieses a todos os profissionais atuantes na equipe de trabalho durante as 24 horas.

Segundo a opinião o autor da mesma pesquisa citada acima, realizada num hospital universitário de Curitiba, a existência de apenas uma anotação de enfermagem em cada turno é insuficiente frente as diversos 
acontecimentos que podem ocorrer neste período(3). Diante do perfil dos clientes atendidos em UTIs, sujeitos a mudanças contínuas no estado de saúde, faz-se necessário realizar anotações com mais freqüência ${ }^{(4)}$.

Considerando que a instituição conta com vínculo de profissionais que trabalham seis horas diárias com plantões alternados em finais de semana, os registros realizados em sua UTI permitem que o responsável pelo cuidado de um turno transmita informações acerca dos acontecimentos em seu período, de maneira fidedigna, quando realizado corretamente. Desta forma, assegura continuidade do processo de trabalho multiprofissional garantindo comunicação efetiva, segurança para o paciente e respaldo do ponto de vista legal e ético ${ }^{(11)}$.

Considerando o processo de enfermagem como ferramenta científica para a garantia da qualidade do cuidado, o enfermeiro deve executar a prescrição de enfermagem sua tomada de decisões, definindo cuidados e orientando a equipe na execução dos $\operatorname{mesmos}^{(5)}$. E, a partir deste modo sistematizado, permite-se a execução de anotações de forma a garantir a continuidade das informações ${ }^{(12)}$.

Segundo os resultados, a primeira coleta permitiu ao enfermeiro a identificação da realização efetiva das ações orientadas por ele, uma vez que os cuidados realizados foram registrados, como forma de respostas dos membros da equipe de enfermagem às solicitações, na prescrição de enfermagem.

Ao se avaliar a qualidade do cuidado, sabe-se que as anotações de enfermagem constituem um indicador fundamental na avaliação da qualidade do cuidado. Porém apenas a freqüência em sua execução não é suficiente para demonstrar efetividade do cuidado(4). Faz-se fundamental que as anotações acerca do atendimento prestado sejam claras e completas ${ }^{(12)}$. Ainda que se tenha obtido valores satisfatórios quanto à existência de anotações de enfermagem evidenciou-se inadequações no que tange a observação de sinais e sintomas condizentes com a patologia e sinais. Pode-se atribuir como fatores causais para estes resultados, o desconhecimento por parte dos enfermeiros e técnicos de enfermagem acerca da ideal qualidade dos registros ou ainda, a incorporação, em sua prática, do exame físico(13).

O exame físico permite o levantamento de informações que devem ser registradas como forma de continuidade de um cuidado individualizado, maneira tal que possibilita conhecimento da situação de saúde do cliente por toda a equipe multiprofissional, detecção de novos problemas, implementação de novas terapia e a partir disto, comparação das respostas aos cuidados prestados $^{(14) .}$

A queda nos percentuais relativos a utilização de corretor líquido e presença de rasuras denota inadequações no que tange a estética textual e possibilidade de entendimento do que se pretende transmitir. Não basta que existam registros de enfermagem. Faz-se necessário que atenda aos critérios de compreensão textual(4).

Pesquisa realizada em um hospital privado da cidade de São Paulo privado revelou que os registros nos prontuários estavam presentes em 143 (95,5\%), enquanto que em 7 (4,5\%) estavam ausentes. Dos registros existentes, 139 (93\%) eram claros e objetivos, 114 (76\%) registros eram precisos, 150 (100\%) registros eram legíveis e 144 (96\%) registros não apresentavam rasuras ${ }^{(11)}$.

Além de impedir legibilidade do texto e uma compreensão eficaz por parte da equipe de saúde, a presença de rasuras nas anotações de enfermagem, independente da forma como é realizada, pode acarretar problemas legais e gerar riscos ao paciente(3).

De forma geral, independente da maneira como o registro é executado, faz-se necessário que disponibilize informações objetivas, fidedignas, corretas e completas a respeito da assistência prestada(4).

Após a execução o cuidado orientado e elaboração de um registro de enfermagem, é indispensável que se proceda a checagem dos itens prescritos e a identificação de quem implementou as ações da assistência de enfermagem, redigindo o nome, função e o número inscrição no Conselho Regional de Enfermagem (COREN). Mesmo existindo legislações que determinam tal procedimento, os profissionais atuantes na unidade passaram por um processo de declínio de sua execução.

Uma pesquisa demonstra que, em relação à identificação do autor dos registros, a maioria é executada de maneira incompleta com 80 (53\%) registros enquanto 18 (12\%) não apresentavam identificação (ausentes) ${ }^{(11)}$. 
Em pesquisa realizada em um hospital universitário da cidade de Curitiba, Paraná, identificou que, de 140 anotações, 120 apresentavam nomes, sendo que alguns se encontravam ilegíveis; em torno de 110 continham a categoria profissional e número do $\operatorname{COREN}^{(3)}$.

Os resultados relativos a não existência de checagem dos itens da prescrição apontam para um viés na qualidade da assistência prestada e na segurança do paciente, uma vez que quando realizada permite identificar se foi realizado e quem realizou, bem como fornecer respaldo ético-legal(3).

Outros estudos apresentam o mesmo problema a exemplo da pesquisa realizada Curitiba, já apresentada nesta discussão, que constatou a maioria dos itens da prescrição sem checagem em duas unidades estudadas. Considerando o caráter privado que possui a instituição citada, o não cumprimento da checagem gerou 21 glosas parciais em 36 contas hospitalares, atribuindo ônus à instituição(3). No contexto das glosas hospitalares permite-se conceituá-las como o cancelamento parcial ou total do orçamento ou itens que o auditor do plano de saúde não considera cabíveis ao orçamento(15).

As anotações de enfermagem redigidas objetiva e criteriosamente garante condições favoráveis a assistência prestada, uma vez que proporciona validade das informações quanto aos aspectos éticos e legais, menores ônus financeiros e ainda, retrata as atividades executadas pela enfermagem faz no atendimento ao cliente. Neste contexto, funciona como uma medida que garante visibilidade à enfermagem ${ }^{(16)}$.

\section{REFERÊNCIAS}

1. Nepomuceno LMR, Kurcgant P. Uso de indicador de qualidade para fundamentar programa de capacitação de profissionais de enfermagem. Rev. esc. enferm. USP. 2008;42(4):665-72. 2. Venturini DA, Marcon SS. Anotações de enfermagem em uma unidade cirúrgica de um hospital escola. Rev. bras. enferm. 2008;61(5):570-75.

3. Luz A, Martins AP, Dynewicz AM. Características de anotações de enfermagem encontradas em auditoria. Rev. Eletr. Enf. [Internet]. 2007 [cited 2012 sep 30];9(2):344-61. Available from: http://www.fen.ufg.br/revista/v9/n2/v9n2a05.htm.

4. Matsuda LM, Carvalho ARS, Évora YDM. Anotações/registros de enfermagem em um hospital-escola. Rev. ciênc. cuid e súde. 2007;6(2):337-46.

5. Vasconcelos MM, Gribel EB, Morais IHSL. Registros em saúde: avaliação da qualidade do prontuário do paciente na atenção básica. Cad. saúde pública. 2008;24 (Suppl 1):S173-S82.

6. Matsuda LM, Silva DMP, Évora YDM, Coimbra JAH.

Anotações/registros de enfermagem: instrumento de comunicação para a qualidade do cuidado? Rev. Eletr. Enf. [Internet]. 2006 [cited

\section{CONCLUSÃO}

Com os resultados apresentados foi possível identificar diversas inadequações relativas ao processo de implementação do cuidado de enfermagem, avaliados a partir da auditoria retrospectiva dos documentos de enfermagem da Unidade de Terapia Intensiva da instituição em estudo.

O Processo de Enfermagem, por si só determina que, após as atividades do cuidado, anotações de enfermagem sejam realizadas de maneira a permitir troca de informações e segurança no processo terapêutico do cliente. As unidades de terapia intensiva, com seus altos investimentos para a recuperação da saúde do cliente, induz a envolver diversos profissionais que compõe a equipe multiprofissional. Estes, por sua vez, devem estar interligados por meio de uma comunicação eficaz e fidedigna, na maioria das vezes, escrita.

Considerando que a qualidade das anotações não é alcançada apenas com a mera execução a cada período de trabalho, mas que envolve a importância de um conteúdo fidedigno, correto e completo, a análise deste estudo permite inferir a necessidade de aperfeiçoamento relativo à execução de anotações de enfermagem com valor legal e assistencial assim como, ao incentivo para incorporação, por parte dos profissionais, dos preceitos do processo de enfermagem.

Desta forma, este trabalho permite suscitar medidas de intervenções no setor em estudo, por meio de processos educativos, visando o aprimoramento das atividades assistenciais.

2012 sep 30];8(3):415-21. Available from: http://www.fen.ufg.br/revista/revista8 3/v8n3a12.htm 7. Feldman L. Avaliação do Serviço de Enfermagem: construção de critérios para análise do serviço. In: Malagutti W, Caetano KC. Gestão do Serviço de Enfermagem no Mundo Globalizado. Rio de Janeiro: Rubio; 2009. p. 29-34.

8. Cianciarullo TI, Gualda DMR, Melleiro MM, organizadoras. Indicadores de qualidade: uma abordagem perinatal. São Paulo: Ícone; 1998.

9. Cianciarullo TI. C \& Q: teoria e prática em auditoria de cuidados. São Paulo: Ícone; 1997.

10. Haddad MCL. Qualidade da assistência de enfermagem: o processo de avaliação em hospital universitário público [thesis]. Ribeirão Preto: Escola de Enfermagem/USP; 2004. 201 p. 11. Draganov PB, Reichert MCF. Avaliação do padrão dos registros de enfermagem em um hospital privado na cidade de São Paulo. Rev. enferm. UFPE on line. 2007 [cited 2012 sep 30];1(1):36-45. Available from: http://www.ufpe.br/revistaenfermagem/index.php/revista/article/ viewFile/10/pdf 165 
12. Carrijo AR, Oguisso T. Trajetória das anotações de Enfermagem: um levantamento em periódicos nacionais (1957-2005). Rev. bras. enferm. 2006;56(esp):454-48.

13. Costa SP, Paz AA, Souza EN. Avaliação dos registros de enfermagem quanto ao exame físico. Rev. Gaúcha Enferm., Porto Alegre (RS). 2010;31(1):62-9.

14. Fernandes AP, Vancini CR, Cohrs F, Moreira RSL. Qualidade das anotações de enfermagem relacionadas à ressuscitação cardiopulmonar comparadas ao modelo Utstein Acta Paul Enferm 2010;23(6):757-63.

15. Ferreira TS, Souza-Braga AL, Cavalcanti-Valente GS, Souza DF, Carvalho-Alves EM. Auditoria em enfermagem: impacto das anotações de enfermagem no contexto das glosas hospitalares. Rev. Aquichan, Colômbia. 2009;9(1): 38-49.

16. Camelo SHH, Pinheiro A, Campos D, Oliveira TL. Auditoria de enfermagem e a qualidade da assistência à saúde: uma revisão da literatura. Rev. Eletr. Enf. [Internet]. 2009 [cited 2012 sep

30];11(4):1018-25. Available from:

http://www.fen.ufg.br/fen_revista/v11/n4/pdf/v11n4a28.pdf.

Artigo recebido em 13/03/2011.

Aprovado para publicação em 28/03/2012.

Artigo publicado em 30/09/2012. 